\title{
Spectrum Sensing for Cognitive Radio Using Hybrid Matched Filter Single Cycle Cyclostationary Feature Detector
}

\author{
Divya Joshi ${ }^{1}$ and Neeru Sharma ${ }^{2}$ \\ ${ }^{1,2}$ Jaypee University of Information Technology, ECE Deptt.Waknaghat,Solan -173234, India \\ E-mail: ${ }^{1}$ divyajoshi26@gmail.com, ${ }^{2}$ neeru.sharma@juit.ac.in \\ Jaskirat Singh $^{3}$ \\ ${ }^{3}$ Jaipur Engineering College \& Research Center,Jaipur-303905, India \\ E-mail: jsingh6@lakeheadu.ca
}

\begin{abstract}
Spectrum sensing is an important task in cognitive radio (CR). Matched filter technique one of the techniques employed for spectrum sensing in cognitive radio which faces the challenge of low frequency offset tolerance in very low SNR environments. Hybrid matched filter architecture is used to improve this frequency offset tolerance. But, overall even such kind of architecture results in coarse detection. In very low SNR environments, where primary user is highly mobile, the multipath profile results in unknown phase of signal. Such kind of signal cannot even be detected by Hybrid Matched Filter. In this paper we propose combination of Hybrid Matched Filter and Single Cycle Cyclostationary Feature Detector to enhance the detection of such architecture. This results in both high frequency offset tolerance as well as fine detection of signal with unknown phase in very low SNR environments. Simulation results show significant improvement in the probability of detection and false alarm of the proposed scheme over Hybrid Matched Filter.
\end{abstract}

Index Terms-Cognitive Radio, Spectrum Sensing, Matched Filter Detection, Hybrid Matched Filter, Single Cycle Cyclostationary Detection.
Wireless communication is a revolution in life of mankind. Innovative services and higher data rates are provided by new wireless devices. Both the licensed and unlicensed spectrum is available for different wireless services. Because of the exponential increase in wireless devices and their usage, the unlicensed spectrum is becoming scarce. Federal Communication Commission (FCC) report says that beyond $70 \%$ of radio spectrum is underutilized [1]. Cognitive Radio (CR) is the key to exploit this underutilized spectrum. The concept of cognitive radio was first coined by Joseph Mitola III in 1999 [2]. The idea of cognitive radio provides a solution by which efficient utilization of the spectrum is possible by applying the spectrum sharing techniques. Cognitive Radio is a radio or system which has the adequacy of sensing its operative electromagnetic surroundings to find spectrum fortuity, employing the underutilized spectrum (spectrum holes) to disseminate anywhere and at any time needed and hence improving spectrum utilization. CR is based on the collaboration of Software Defined Radio (SDR) and artificial intelligence which are the two main components for assuring efficient use of underutilized spectrum [2][3]. Spectrum hole or white space is unused spectrum when the licensed user is idle, as shown in left portion of Fig.1. The right portion of Fig. 1 shows the cognitive radio transceiver architecture.

\section{INTRODUCTION}
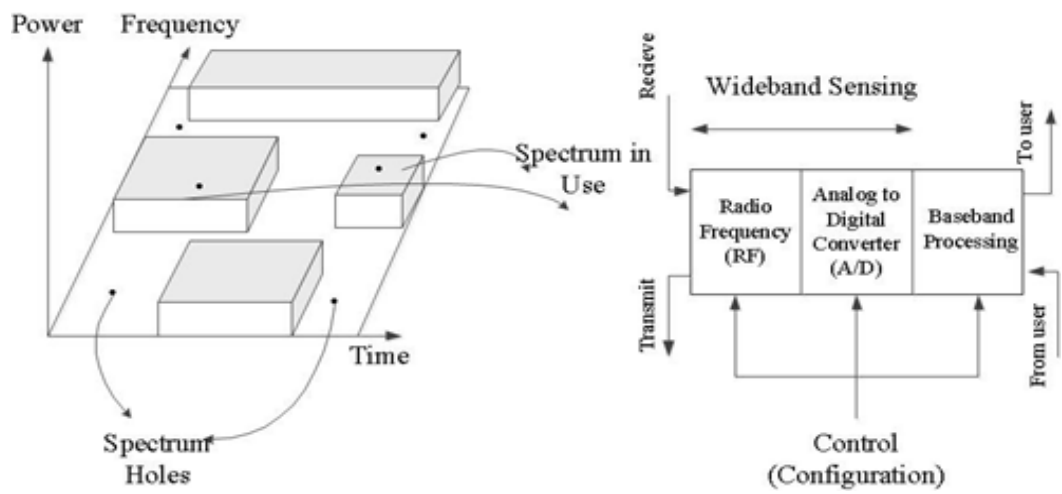

Fig. 1. Overview of spectrum hole concept and cognitive radio transceiver architecture 
The main objectives of cognitive radio are as follows:

1. Spectrum sensing: Determining available spectrum holes and revealing the presence of licensed users [4].

2. Spectrum management: Making the adaptability of the available spectrum, huge [5].

3. Spectrum sharing: Providing moderate spectrum scheduling to already allocate unlicensed users.

4. Spectrum mobility: Prolong consistent transition from one spectrum hole to another.

The success of cognitive radio largely depends upon the competency of the first step of spectrum sensing in the cognitive radio cycle [4]. The spectrum sensing technique deployed by CR can be classified broadly into Non- cooperative detection (Transmitter detection), Interference based detection and Cooperative detection. Furthermore, Non-cooperative detection is classified into: Matched Filter Detection (MFD), Energy Detection (ED) and Cyclostationary Feature Detection (CFD). The focus of this paper is on Hybrid Matched Filter detection which is a kind of MFD scheme and belongs to the class of Non-cooperative sensing schemes [6]. Section II discusses the Hybrid Matched Filter (HMF) [6] and Single cycle Cyclostationary Detector [7]. In section III, the proposed work is explained. Simulation results are discussed in Section IV. Paper is concluded in section V.

\section{RELATED WORK}

Spectrum sensing technique facilitates the efficient usage of the available spectrum. Various spectrum sensing methods are proposed in literature. [13] defines cognitive radio channel as a two-sender, two-receiver interference channel in which sender two obtains the encoded message sender one transmits. [14] considers the sensing duration to maximize the throughput for the secondary network. Energy detection scheme is used here to find the optimal sensing time. [15] explains cooperative sensing and its various forms. Also external sensing algorithms and other sensing methods are discussed. Databases and geolocation has been considered by using beacons for spectrum sensing.

In [16], the benefits of cooperation in cognitive radio are illustrated. Authors have shown that by allowing the cognitive users operating in the same band to cooperate the detection time can be reduced and thus increase the overall agility. In [17], a linear cooperation framework has been proposed. Interference to primary radio was minimized and spectrum utilization was increased. In
[18], two sensing algorithms are suggested, one is based on the ratio of the maximum eigen value to minimum eigen value; the other is based on the ratio of the average eigen value to minimum eigen value. In [19], the cognitive radio users were made to cooperate with each other in trying to detect licensed transmissions. In [20], wideband spectrum ensing technique, called multiband joint detection, which jointly detects the signal energy levels over multiple frequency bands rather than consider one band at a time. In [21], hybrid channel state acquisition schemes were considered that used cooperative multiband sensing to build a virtual spectrum database for ad hoc CR systems, which was referred as the spectrum pool.

This technique helps cognitive radio to search for the existence of primary user [4]. In Non-corporative sensing, CR exploits different kinds of features embedded in the signal of primary user to detect its presence. Energy Detection scheme uses sampled energy of the received waveform as the threshold to determine the presence of primary user [4]. The basic operation of Matched Filter is similar to the correlation function in which the signal is convolved with filter whose impulse response is time inverted version of the signal itself [4]. Therefore, it requires prior information about the transmitted waveform by primary user. MFD cannot differentiate between signal and noise below the SNR of $-20 \mathrm{~dB}$. Cyclostationary Feature Detection (CFD) works on the principle that most of the man-made signals are stationary in wide sense, that is one or more of their statistics vary periodically with time [8]. Such kinds of signals are called Cyclostationary or Polycyclostationary depending on whether the statistics vary with single or multiple periodicities respectively [8]. The most commonly used statistic is the mean of the signal. Thus, if any of the statistics of the received signal is varying with either single or multiple periodicities then the primary user signal is present.

\section{A. Hybrid Matched Filter}

It has been proved in [4][6] that Matched Filter Detection (MFD) has the fastest sensing time among all the non-cooperative schemes till a certain low threshold value of Signal-to-Noise Ratio (SNR). After this value, sensing time increases in an unbounded fashion [9]. Also, MFD faces another problem of low frequency offset tolerance in multipath fading scenarios which renders it useless for low SNR environments [6]. Therefore, Hybrid Matched Filter (HMF) has been proposed in the literature as a solution to this problem, which has been shown in Fig. 2 [6]. 


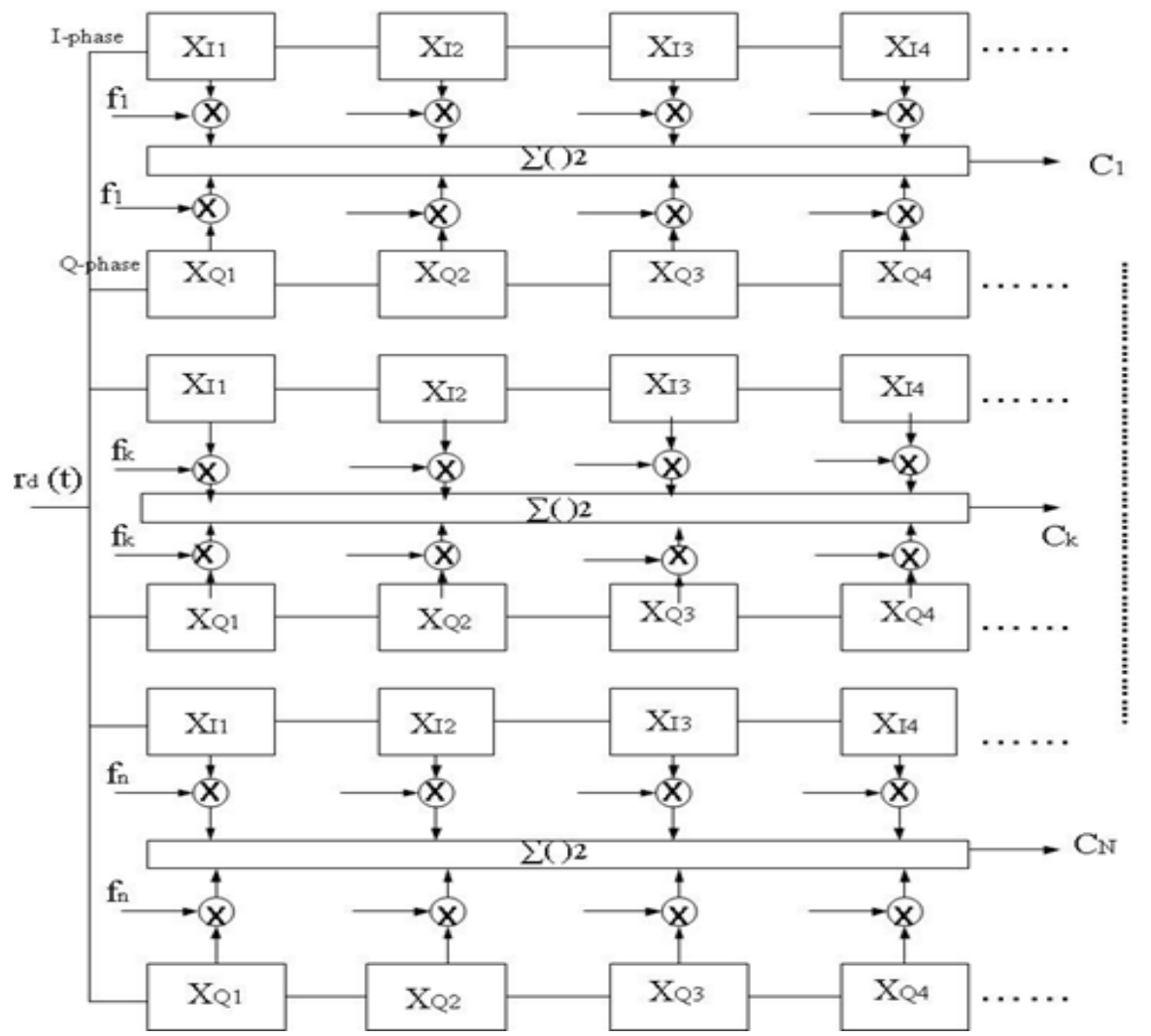

Fig.2. Structure of hybrid matched filter detector

HMF is the combination of orthogonal, parallel and segmented matched filter where each parallel branch demodulates the received signal at different residual offset frequency and there are several segments [6]. Furthermore, each segment is an orthogonal matched filter. Therefore instead of performing sequential checks on different residual frequencies to identify the correct offset, each parallel branch performs parallel check at the same time which enhances the sensing time [6][10][11]. If $f_{\min }$ is the minimum frequency offset tolerance of a matched filter then the desired frequency offset tolerance, $f_{T O}$ is given by [6][10][11]:

$$
f_{T O}=N_{s e g} \times f_{\min }
$$

Where $N_{\text {seg }}$ is the number of segments in one parallel branch of HMF [10][11].

Let $N$ be the number of samples of a transmitted waveform taken over one period during sampling. When the received signal is demodulated to baseband frequency and sampled at a certain frequency $f_{s}$, and then the sensing time is given by $N / f_{s}$ [6][9][12]. The sampling frequency is given by:

$$
f_{s}=N_{\text {seg }} \times N_{s} \text { samples/sec }
$$

Where $N_{s}$ is the number of samples per segment [11].Thus, increasing the sampling frequency has a twofold benefit. It increases the frequency offset tolerance because increasing the sampling frequency leads to increase in $N_{\text {seg }}$ which will increase $f_{T O}$ as can be seen from (1). The sensing time can also be improved by over sampling at the receiver by increasing the number of samples [6][12]. Once the number of segments is fixed in order to acquire a desired frequency offset tolerance, then number of samples can be further increased by increasing the number of samples per segment [6]. Therefore, HMF also results in reduced sensing time in low SNR scenarios along with high frequency offset tolerance by deploying different parallel branches each working on certain residual offset frequency [6][10].

\section{B. Single Cycle Cyclostationary Feature Detector}

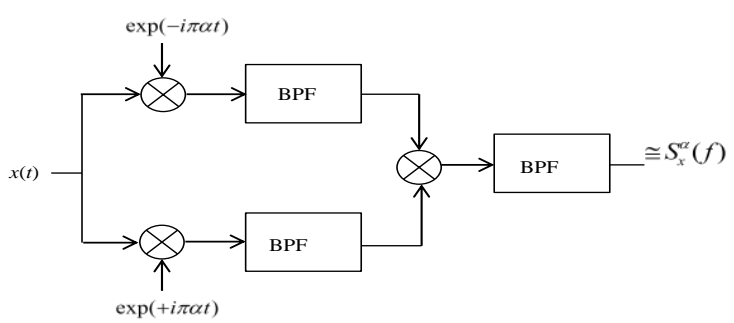

Fig. 3. Single cycle cyclostationary detector

Gardner's definition of cyclostationarity says that a signal is said to have cyclostationarity if the mean value of its second order delay product exhibits periodicity. The mean value of second order delay product is also 
called autocorrelation function [7][8]. Fig. 3 shows the single cycle cyclostationary detector. There are two approaches for the cyclostationary analysis of a signal: Stochastic as well as non-stochastic approach [8]. In this paper, focus is on stochastic approach where the received signal is modeled as a stochastic process having several sample functions. The autocorrelation function is given by:

$$
R_{X}(\tau)=\mathrm{E}[X(t) X(t+\tau)]
$$

where, $\mathrm{E}[\cdot]$ is the expected value operator which is the mean value equivalent in probability space theory [8]. Furthermore, $X(t)$ and $X(t+\tau)$ are the two random variables at time instants $t$ and $(t+\tau)$. Now, if the received signal exhibits cyclostationarity then the probability space autocorrelation function will be periodic in behaviour [7][8]. Since, it is known that any periodic function can be represented using Fourier series, therefore, the probability space autocorrelation function is given as [7][8]:

$$
R_{X}^{\{\alpha\}}=\sum_{n=-\infty}^{\infty} R_{X}^{\alpha} e^{j n \alpha t}=R_{X}(\tau)
$$

where, $R_{X}^{\alpha}$ are the Fourier coefficients of individual components at different cyclic frequencies, $\alpha$. Furthermore, these Fourier coefficients are equal to the Cumulative Distribution Functions (CDFs) evaluated at different sampling instants [8] and hence we can re-write the above equation as:

$$
F_{X}^{\{\alpha\}}=\sum_{n=-\infty}^{\infty} F_{X}^{\alpha} e^{j n \alpha t}=R_{X}^{\{\alpha\}}
$$

where, $F_{X}^{\alpha}$ is a Cumulative Distribution Function which is defined as [7][8]:

$$
F_{X}^{\alpha}=\operatorname{Prob}[X(t+\tau)<x]
$$

Therefore, probability space autocorrelation function can also be found out by evaluating Cumulative Distribution Function at every sample of a sampled signal [8]. If this autocorrelation function is periodic or polyperiodic then it is called cyclic autocorrelation function and shows the presence of a signal [8]. The stochastic approach to detect any cyclostationary signatures inside the received signal has been discussed in this section. This will act as a background to accomplish the signal processing operations involved in our proposed work.

\section{PROPOSED WORK}

The desired frequency offset tolerance of Hybrid matched filter (HMF) is given by (1). Therefore, total number of samples per parallel branch of HMF is given by $f_{s}=N_{\text {total }}=N_{s} \times N_{\text {seg }}$, where $N_{\text {total }}$ is the total number of samples per parallel branch of HMF and $N_{s}$ is the number of samples per segment. Let's say that the transmitted signal is given by:

$$
m(t)=c(t) \sqrt{2} S \cos \left(2 \pi f_{b} t+\phi_{M}\right)
$$

Where, $c(t)$ is the code word waveform which is modulated over one of the M-ary PSK waveform, Sis the signal power, $f_{b}$ is the baseband frequency, $\phi_{m}$ is one of the values of M-ary phase. But because of channel impairments such as Doppler shift and Additive White Gaussian Noise (AWGN), the receiver will receive $m(t)$ in the form given below:

$$
r(t)=c(t) \sqrt{2} S \cos \left(2 \pi f_{b} t+\phi_{M}+\theta(t)\right)+n(t)
$$

where, $\theta(t)$ is a dynamic phase due to Doppler shift which is the function of time. $n(t)$ is the AWGN process. Therefore, the received signal can be represented as:

$$
r(t)=c(t) \sqrt{2} S \cos \left(2 \pi f_{b} t+\varphi(t)\right)+n(t)
$$

Where, $\varphi(t)=\phi_{M}+\theta(t)$ and is the total dynamic phase shift.

The received signal $r(t)$ is then demodulated at different residual offset frequencies whose number is equal to the number of parallel branches in $\mathrm{HMF}$ structure, where each branch samples $r(t)$ into $N_{\text {total }}$ samples. Therefore, the demodulated received signal in branch $i, r_{d}^{i}(t)$ is given by:

$$
r_{d}^{i}(t)=r(t)\left[\cos \left(2 \pi\left(f_{b}-f_{r i}\right) t+\phi_{M}\right)+j \sin \left(2 \pi\left(f_{b}-f_{r i}\right) t+\phi_{M}\right)\right]
$$

Where, $f_{r i}$ is the residual offset frequency of branch $i$. Furthermore, signal can be rewritten as:

$$
r_{d}^{i}(t)=\left[r_{I}^{i}(t)+n_{I}^{i}(t)\right]+j\left[r_{Q}^{i}(t)+n_{Q}^{i}(t)\right]
$$

Where, $r_{I}^{i}(t)$ is the I-phase component of the received demodulated signal, $n_{I}^{i}(t)$ is I-phase component of the AWGN process, $r_{Q}^{i}(t)$ is the Q-phase component of the received demodulated signal and $n_{Q}^{i}(t)$ is the Q-phase component of AWGN process.

$$
\begin{aligned}
& r_{I}^{i}(t)=c(t) \sqrt{2} S \cos \left[2 \pi f_{b} t+\varphi(t)\right] \cdot \cos \left[2 \pi\left(f_{b}-f_{r i}\right) t+\phi_{M}\right] \\
& r_{Q}^{i}(t)=c(t) \sqrt{2} S \cos \left[2 \pi f_{b} t+\varphi(t)\right] \cdot \sin \left[2 \pi\left(f_{b}-f_{r i}\right) t+\phi_{M}\right]
\end{aligned}
$$


Similarly, the I-phase and Q-phase components, $n_{I}^{i}(t)$ and $n_{Q}^{i}(t)$ respectively of AWGN process are given as:

$$
\begin{aligned}
& n_{I}^{i}(t)=n(t) \cos \left[2 \pi\left(f_{b}-f_{r i}\right) t+\phi_{m}\right] \\
& n_{Q}^{i}(t)=n(t) \sin \left[2 \pi\left(f_{b}-f_{r i}\right) t+\phi_{m}\right]
\end{aligned}
$$

The received demodulated signal $r_{d}^{i}(t)$ is sampled at sampling instants of $T_{s}=1 / N_{\text {total }}$ which results in column vectors. Therefore,

$$
r_{d}^{i}\left(T_{s}\right)=\left[r_{I}^{i}\left(T_{s}\right)+n_{I}^{i}\left(T_{s}\right)\right]+j\left[r_{Q}^{i}\left(T_{s}\right)+n_{Q}^{i}\left(T_{s}\right)\right]
$$

where, every element is a column vector. Furthermore,

$$
r_{d}^{i}\left(T_{s}\right)=\left[X_{I}^{i}\left(t+n T_{s}\right)\right]+j\left[X_{Q}^{i}\left(t+n T_{s}\right)\right]
$$

where, $X_{I}^{i}\left(t+n T_{s}\right)$ and $X_{Q}^{i}\left(t+n T_{s}\right)$ are the column vectors of Gaussian distributed random variables with mean as the corresponding elements of the column vectors $r_{I}^{i}\left(T_{s}\right)$ and $r_{Q}^{i}\left(T_{s}\right)$ respectively and the variance is set according to desired SNR. Then the magnitude squared values of the statistic to be observed is calculated for every branch $i$ as:

$$
M_{i}=\sum_{n=1}^{N_{\text {total }}}\left[X_{I}^{i}\left(t+n T_{s}\right)\right]^{2}+\sum_{n=1}^{N_{\text {total }}}\left[X_{Q}^{i}\left(t+n T_{s}\right)\right]^{2}
$$

This statistic results in a column vectors, as for every single cyclic shift of codeword by one sample, one element of $M_{i}$ will be produced. Therefore, $V$ will be two dimensional vector such that $V=\left[M_{1} M_{2}\right.$

where $B$ is the total number of parallel branches and $M_{i}$ is a column vector. Therefore, after a complete cyclic shift at every branch, the output will be a row vector of normalized correlation coefficients, $C=\left[c_{1} c_{2} c_{3} \ldots \ldots \ldots c_{B}\right]$ calculated from individual columns of two dimensional vector $V$. Every element of $C$ is the correlation coefficient of the corresponding branch. Hence, the received demodulated signal of branch jis selected for further processing if $c_{j}=\max \left\{c_{i}\right\}$ where $i \neq j$.

Furthermore, the autocorrelation function, $R_{j}^{\{\alpha\}}(\tau)$ of received demodulated signal of branch $j$ is given by:

$$
R_{j}^{\{\alpha\}}(\tau)=F_{j I}^{\{\alpha\}}\left[N\left(\mu_{j I}, \sigma_{j I}^{2}\right)\right]+i F_{j Q}^{\{\alpha\}}\left[N\left(\mu_{j Q}, \sigma_{j Q}^{2}\right)\right]
$$

where, $F_{j I}^{\{\alpha\}}$ is the Cumulative Distribution Function evaluated for the Normal Distribution $N\left(\mu_{j I}, \sigma_{j l}^{2}\right)$ and $F_{j Q}^{\{\alpha\}}$ is the Cumulative Distribution Function evaluated for the Normal Distribution $N\left(\mu_{j Q}, \sigma_{j Q}^{2}\right)$. The $\{\alpha\}$ in the suffix shows that it is a set which can consist of one cyclic frequency or multiple cyclic frequencies. If it consists of only one cyclic frequency as zero then primary user signal is not present. If it consists of only one cyclic frequency then primary user signal is present and cyclostationary otherwise polycyclostationary. The degree of similarity between transmitted signal and received signal is further determined by computing magnitude squared autocoherence (MSAC) value, $M S A C_{j}$ which is given by:

$$
M S A C_{j}=\frac{|M(f)|^{2}}{\left|R_{j}^{\{\alpha\}}\left(f-\frac{\alpha}{2}\right)\right|\left|R_{j}^{\{\alpha\}}\left(f+\frac{\alpha}{2}\right)\right|}
$$

where, $M(f)$ is the power spectral density of the transmitted signal. In order to perform hypothesis test on $M S A C_{j}$, the probability distribution followed by these values is to be determined by performing Monte Carlo simulation for large number of times.

Hypothesis test is further performed on that distribution with null hypothesis stating that only noise is present, whereas alternative hypothesis stating that primary user signal is present with a specific level of significance.

\section{SimUlation ANALYSIS AND RESULTS}

A set of extensive simulations have been performed for both HMF and the proposed scheme on Matlab. The performance is evaluated in terms of probability of detection and probability of false alarm. The prominence is to evaluate the comparative performance of HMF and the proposed scheme. In the performed simulations, the number of samples per segment has been set to four.

To determine the distribution followed by magnitude squared autocoherence value, an extensive set of Monte Carlo simulations for different values of SNR have been performed for 1000 iterations and it came out to be lognormal distribution for the case when primary user signal is present.

During the actual simulations, each computed value of magnitude squared autocoherence have been put through hypothesis test on the log-normal distribution of generated magnitude squared autocoherence values at a significance level of 0.15 . The null hypothesis in the test is the absence of signal whereas the alternative hypothesis is the presence of the signal.

Fig. 4 demonstrates higher probability of detection of the proposed scheme in comparison to the HMF. This is because the algorithm selects the highest value of correlation coefficient from all the corresponding correlation values of individual branch. This highest value is the indicator that the residual offset frequency of the corresponding branch used in demodulating the signal finds perfect correlation with the Doppler phase shifted received signal due to multipath profile. This provides 
the coarse detection that some signal has been detected whose phase shift actually correlates well with one of the residual offset frequencies. Then the demodulated signal using that residual offset frequency is sent to the cascaded stage of processing where it becomes further processed using single cycle Cyclostationary Feature Detector. Therefore, due to this cascaded stage of Cyclostationary Feature Detector, the course detection becomes finer; hence there is little chance of being a miss detection.

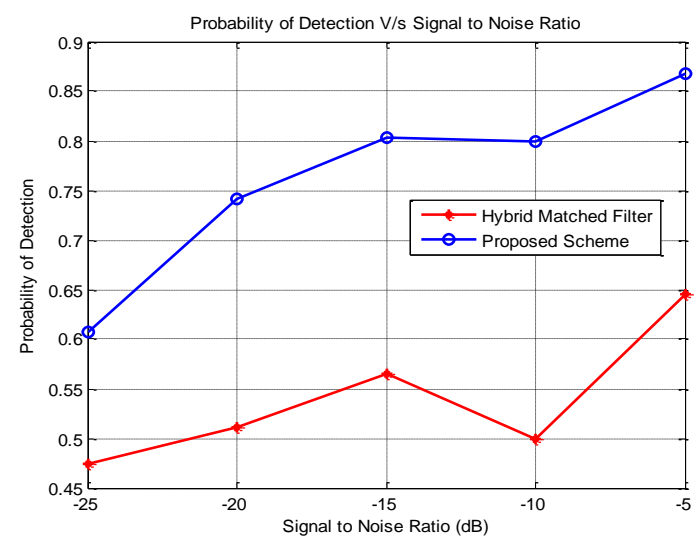

Fig. 4. Probability of detection for combined hybrid matched filter single cycle cyclostationary detection

Since, the probability of detection increases therefore, the probability of false alarm reduces. Fig. 5 depicts the same trend in the probability of false alarm for the proposed scheme as well as HMF. The probability of false alarm for proposed scheme is much less than that of HMF.

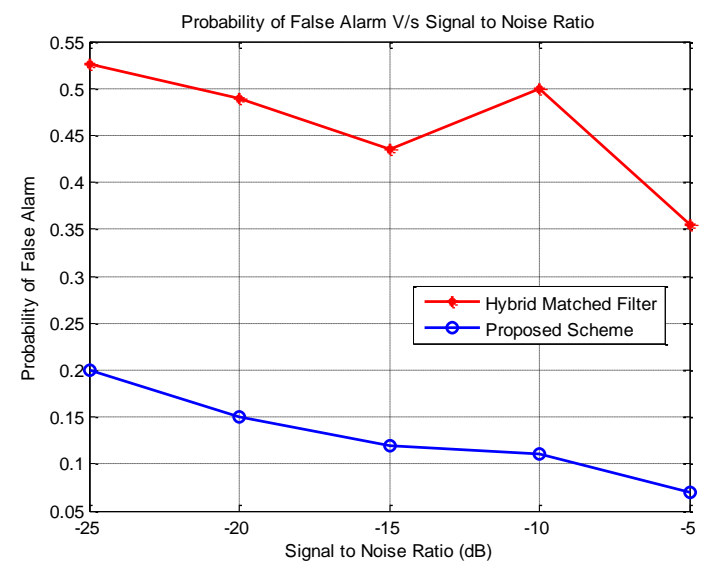

Fig. 5. Probability of false alarm for combined hybrid matched filter single cycle cyclostationary detection

\section{CONCLUSION}

Simulation results depict significant improvement in the probability of detection and probability of false alarm in case of proposed scheme over HMF. Hence, the proposed scheme clearly outperforms HMF in terms of both metrics. But the proposed scheme incurs significant overhead in terms of execution time involved in the signal processing related to cascaded stages. Therefore, it would be interesting to reduce the execution time of the proposed scheme.

\section{REFERENCES}

[1] Federal Communication Commission, "Spectrum Policy Task force report", ET Docket No. 02-135, Nov. 2002.

[2] Mitola, J. and J. Maguire, G. Q., "Cognitive radio: making software radios more personal." IEEE Personal Commun. Mag., vol. 6, no. 4, Aug. 1999, pp. 13-18.

[3] S. Haykin, "Cognitive radio: brain-empowered wireless communications," "IEEE J. Select. Areas Commun.", vol. 23, no. 2, 2005, pp. 201-220.

[4] Ian F. Akyildiz, Won-Yeol Lee, Mehmet C. Vuran, "NeXt generation/dynamic spectrum access/ cognitive radio wireless networks: A survey", Computer Networks 50(2006), p2127-2159(2006).

[5] I. F. Akyildizet al., "A Survey on Spectrum Management in Cognitive Radio Networks," IEEE Commun. Mag., vol. 46, no. 4, Apr. 2008, pp. 40-48.

[6] Zhang Z, Yang Q, Wang L, Zhou X (2010), "A novel hybrid matched filter structure for IEEE 802.22 standard". Circuit Syst (APCCAS), IEEE Asia Pacific conference, 2010, pp 652-655

[7] S. Enserink and D. Cochran, "A cyclostationary feature detector," in Proc. 28th Asilomar Conf. Signals, Systems, Computers, pp. 806-810, October, 1994. Article (CrossRef Link).

[8] W.A. Gardner, "Exploitation of spectral redundancy in cyclostationary signals," IEEE Signal Processing Magazine, vol. 8, no. 2, pp. 14-36, April 1991.

[9] D. Cabric, "Addressing feasibility of cognitive radios," IEEE Signal Process. Mag., vol. 25, no. 6, pp. 85-93, Nov. 2008.

[10] M.S. Rahaman, D.E. Rodds, "Throughput and PN Codephase Acquisition for Packet CDMA without Preamble"

[11] B. Persson, D.E. Dodds, and R.J. Bolton, "A Segmented Matched Filter for CDMA Code Synchronization in Systems with Doppler Frequency Offset" Proceedings IEEE Globecom, San Antonio, Texas, Nov 2001.

[12] P. K. Sagiraju, S. Agaian, and D. Akopian, "Reduced complexity acquisition of GPS signals for software embedded applications," IEE Proc.-Radar Sonar Navig., vol. 153, no. 1, pp. 69-78, Feb. 2006, doi: 10.1049/iprsn:20050091

[13] Natasha Devroye, Patrick Mitran, "Achievable Rates in Cognitive Radio Channels", IEEE Transactions on Information Theory, vol. 52, no. 5, may 2006, pp. 18131827.

[14] Ying-Chang Liang, Yonghong Zeng, "SensingThroughput Tradeoff for Cognitive Radio Networks", IEEE Transactions on Wireless Communications, vol. 7, no. 4, April 2008, pp 1326-1337.

[15] Tevfik Yucek and Huseyin Arslan, "A Survey of Spectrum Sensing Algorithms for Cognitive Radio Applications", IEEE Communications Surveys \& Tutorials, vol. 11, no. 1, pp. 116-130.

[16] Ghurumuruhan Ganesan and Ye (Geoffrey) Li, "Cooperative Spectrum Sensing in Cognitive Radio,Part I: Two User Networks", IEEE Transactions on Wireless Communications, vol. 6, no. 6, June 2007, pp. 2204-2213.

[17] Zhi Quan, Shuguang Cui and Ali H. Sayed, "Optimal Linear Cooperation for Spectrum Sensing inCognitive Radio Networks", IEEE Journal of Selected Topics in 
Signal Processing, vol. 2, no. 1, February 2008, pp. 28-40.

[18] Yonghong Zeng, Ying-Chang Liang, "Eigenvalue-Based Spectrum Sensing Algorithms forCognitive Radio", IEEE Transactions on Communications, vol. 57, no. 6, June 2009, pp. 1784-1793.

[19] Jayakrishnan Unnikrishnan, Venugopal V. Veeravalli, "Cooperative Sensing for PrimaryDetection in Cognitive Radio", IEEE Journal of Selected Topics in Signal Processing, vol. 2, no. 1, February 2008, pp. 18-27.

[20] Zhi Quan, Shuguang Cui,Ali H. Sayed, "Wideband Spectrum Sensing in Cognitive Radio Networks", IEEE International Conference on Communications, 2008, pp. 901-906.

[21] Woongsup Lee, Dong-Ho Cho, "New Cooperation-Based Channel State Acquisition Scheme for Ad Hoc Cognitive Radio Systems", IEEE Transactions on Vehicular Technology, vol. 62, no. 7, September 2013, pp. 33253338 .

\section{Authors' Profiles}

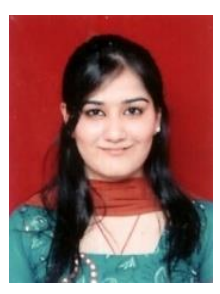

Divya Joshi completed her Master of Technology from the Department of Electronics \& Communication Engineering, Jaypee University of Information Technology, Waknaghat, Solan (H.P.), India in 2014. She did her B.E. in Electronics and Communication Engineering from Maharishi Arvind Institute of Engineering $\&$ Technology, Jaipur, India. Her area of interest is $5 \mathrm{G}$ wireless communication and wireless sensor networks.

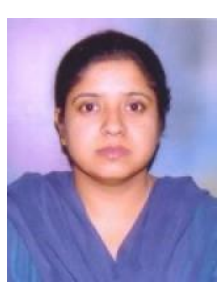

Neeru Sharma is an Assistant Professor (Senior Grade) in the department of Electronics and Communication Engineering, Jaypee University of Information Technology, Waknaghat,Solan, India. She completed her Ph.D in Electronics and Communication Engineering from Jaypee University of Information Technology, Waknaghat, Solan, India. Her area of interest is $3 \mathrm{G}$ and beyond wireless networks.

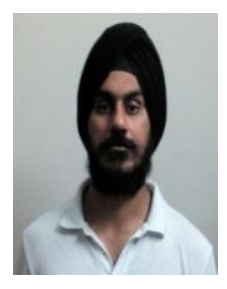

Jaskirat Singh is an Assistant Professor - II in the department of Electronics and Communication Engineering, JECRC University, Jaipur, India. He did his B.Tech in Electronics and Communication Engineering from Sikkim Manipal Institute of Technology, Sikkim Manipal University, Sikkim, India. He did his MSc. Engg. in Electrical and Computer Engineering from Lakehead University, Canada. His research interests include 5G wireless communications and Cognitive Radios.

How to cite this paper: Divya Joshi, Neeru Sharma, Jaskirat Singh,"Spectrum Sensing for Cognitive Radio Using Hybrid Matched Filter Single Cycle Cyclostationary Feature Detector", IJIEEB, vol.7, no.5, pp.13-19, 2015. DOI: 10.5815/ijieeb.2015.05.03 